\title{
Effect of the Energy Window Width on the Estimation of Renal Function from Dynamic Renal Scintigraphy with ${ }^{99 m T c-M A G 3}$
}

\author{
Yuji Asano', Yusuke Ohzeki², Yusuke Inoue ${ }^{*}$ \\ ${ }^{1}$ Department of Diagnostic Radiology, Kitasato University School of Medicine, Sagamihara, Japan \\ ${ }^{2}$ Department of Radiology, Kitasato University Hospital, Sagamihara, Japan \\ Email: ${ }^{\text {inoueys34@gmail.com }}$
}

Received 21 March 2015; accepted 10 May 2015; published 15 May 2015

Copyright (C) 2015 by authors and Scientific Research Publishing Inc.

This work is licensed under the Creative Commons Attribution International License (CC BY). http://creativecommons.org/licenses/by/4.0/

c) (i) Open Access

\begin{abstract}
Objective: The width of the photopeak energy window influences the image quality and quantitative accuracy of gamma camera imaging. We compared $20 \%$ and $15 \%$ energy windows in renal scintigraphy with ${ }^{99 \mathrm{~m} T c-m e r c a p t o a c e t y l t r i g l y c i n e ~(M A G 3), ~ e s p e c i a l l y ~ i n ~ t e r m s ~ o f ~ c a m e r a-b a s e d ~}$ quantitative estimation of renal function. Materials and Methods: Forty patients who underwent dynamic renal scintigraphy with ${ }^{99 m T c-M A G 3}$ were enrolled in this study. Images were acquired simultaneously using two energy windows centered at $140 \mathrm{keV}$ and with widths of $20 \%$ and $15 \%$. Fractional renal uptake was calculated as the ratio of initial renal uptake estimated by patient imaging to injected dose estimated by syringe imaging, and was converted to MAG3 clearance using an empirical equation determined previously from data obtained with a $20 \%$ energy window. Relative function of the right kidney was also assessed. Visual evaluation was performed to compare image quality between the $20 \%$ and $15 \%$ energy windows. Results: Both total kidney MAG3 clearance and relative function of the right kidney were identical between the $20 \%$ and $15 \%$ energy windows. Image quality was also similar irrespective of the energy window width. Conclusions: The camera-based method established using a $20 \%$ energy window is applicable for the estimation of renal function using a $15 \%$ energy window, and data obtained using $20 \%$ and $15 \%$ windows are interchangeable.
\end{abstract}

\section{Keywords}

99mTc-Mercaptoacetyltriglycine (MAG3), Dynamic Renal Scintigraphy, Renal Function, Energy Window, Quantitative Measurement

\footnotetext{
${ }^{*}$ Corresponding author.
}

How to cite this paper: Asano, Y., Ohzeki, Y. and Inoue, Y. (2015) Effect of the Energy Window Width on the Estimation of Renal Function from Dynamic Renal Scintigraphy with ${ }^{99 m}$ Tc-MAG3. Open Journal of Medical Imaging, 5, 37-41. 


\section{Introduction}

Dynamic renal scintigraphy with ${ }^{99 \mathrm{~m}} \mathrm{Tc}$-mercaptoacetyltriglycine (MAG3) allows quantitative estimation of differential renal function using camera-based methods without blood sampling [1]-[5]. Initial renal uptake and injected dose are assessed using a gamma camera to calculate fractional renal uptake, which is converted to an index of renal function using a predefined empirical equation [1]-[4].

In gamma camera imaging, the energy of the gamma ray photon is determined with the pulse-height analyzer. Only when the energy is within the predefined photopeak energy window, the gamma ray photon is regarded as primary photons originating from the radionuclide of interest and is recorded to produce a final image. This energy-based selection reduces the contribution of scattered photons and improves the contrast and sharpness of the resulting image.

The width of the photopeak energy window is one of essential parameters of data acquisition using a gamma camera and may influence image quality and quantitative accuracy [6] [7]. For imaging ${ }^{99 \mathrm{~m}} \mathrm{Tc}$ sources, a $20 \%$ or $15 \%$ energy window is commonly used. A narrow window restricts the contamination of scattered photons' efficiently and may provide a sharp image when sufficient counts are obtained. In contrast, reduced counts may cause degradation of image quality due to a signal-to-noise ratio. To estimate renal function, the injected dose is assessed by imaging an injection syringe, while renal uptake is assessed by patient imaging. Scatter conditions differ between syringe imaging and patient imaging, which may cause variations in estimated fractional renal uptake depending on the energy window width.

In the present study, we compared $20 \%$ and $15 \%$ energy windows in renal scintigraphy with ${ }^{99 \mathrm{~m}} \mathrm{Tc}-\mathrm{MAG} 3$, especially in terms of estimation of renal function. The principal aim was to determine the applicability of camera-based methods established using a $20 \%$ energy window to estimation of renal function from data obtained using a $15 \%$ window.

\section{Materials and Methods}

\subsection{Subjects}

Forty patients $(\mathrm{M}: \mathrm{F}=26: 14$; age $62.9 \pm 13.1$ years, mean $\pm \mathrm{SD})$ who underwent dynamic renal scintigraphy with

${ }^{99 \mathrm{~m}} \mathrm{Tc}-\mathrm{MAG} 3$ for various clinical indications from May 2014 to September 2014 were enrolled in this study. Two patients had only a right kidney, and the other patients had both kidneys. The present study was approved by the institutional review board, and need for informed consent was waived.

\subsection{Imaging Procedures}

Thirty min after oral intake of $300 \mathrm{~mL}$ of water, $200 \mathrm{MBq}$ of ${ }^{99 \mathrm{~m}} \mathrm{Tc}-\mathrm{MAG} 3$ was administered intravenously with the patient in the supine position, and dynamic imaging was performed for $30 \mathrm{~min}$. Although we acquired both anterior and posterior images routinely, posterior images were exclusively used for study purpose. A gamma camera system (BrightViewXCT, Philips Healthcare, Cleveland, $\mathrm{OH}$ ) equipped with a low-energy general purpose collimator was used. This system allowed simultaneous acquisition at different frame rates and using distinct, overlapping energy windows. Images were acquired using two energy windows centered at $140 \mathrm{keV}$ and with widths of $20 \%$ and $15 \%$. For each energy window, two data sets were obtained at different frame rates: one consisted of 803 -s frames followed by 104 15-s frames, while the other consisted of 301 -min frames. The 3-s images were used to quantitatively estimate renal function and visually evaluate perfusion, and the 1-min images were used for visual evaluation of renal function and urinary excretion. A $128 \times 128$ matrix was employed, and the zoom factor was set at 1 .

The injected dose was measured with the same gamma camera system using $20 \%$ and $15 \%$ energy windows. The syringe was placed on the imaging table above the detector head before and after injection, and data were acquired for $10 \mathrm{~s}$ each. A $512 \times 512$ matrix was used, with a zoom factor of 1.46.

\subsection{Estimation of Renal Function}

Regions of interest (ROIs) were drawn for the kidneys and subrenal background areas by a semiautomated method $[8]$ on images acquired with the $20 \%$ energy window, and modified manually if necessary. The ROIs were stored and applied for the analysis of images obtained using the $15 \%$ window. Background-subtracted renal 
counts at $1-2$ min after tracer arrival at the kidney (cpm) were calculated for the right $(C r)$ and left $(C l)$ kidneys.

Renal depth was calculated by the following formulas [9]:

$$
\begin{aligned}
& D r=16.778 \times B W / B H+0.752 \\
& D l=16.825 \times B W / B H+0.397,
\end{aligned}
$$

where $D r$ and $D l$ are the depths (cm) of the right and left kidneys, respectively, $B W$ is body weight $(\mathrm{kg})$, and $B H$ is body height $(\mathrm{cm})$. Attenuation factors for the right $(A F r)$ and left $(A F l)$ kidneys were estimated, assuming an attenuation coefficient of $0.10 / \mathrm{cm}$, as follows:

$$
\begin{aligned}
& A F r=\exp (-0.10 \times D r) \\
& A F l=\exp (-0.10 \times D l) .
\end{aligned}
$$

Counts for the syringe were determined using a square ROI of $206 \times 206$ pixels before and after injection and corrected for decay to the injection time. The injected count $(\mathrm{Ci})$ was calculated as the preinjection count minus the postinjection count and was expressed as cpm.

The percent renal uptake $(\% R U)$ was calculated as follows:

$$
\% R U=100 \times(C r / A F r+C l / A F l) / C i .
$$

The percent renal uptake for the $20 \%$ window was calculated using patient and syringe images obtained with the $20 \%$ energy window, while the percent renal uptake for the $15 \%$ window was calculated using patient and syringe images obtained with the $15 \%$ window.

The MAG3 clearance $(C L)$ was calculated using a conversion equation: $C L=13.577 \times \% R U+0.50$. This equation was determined empirically based on data obtained using a $20 \%$ energy window in a previous study [4]. In the present study, the same equation was used for the $20 \%$ and $15 \%$ windows. Relative renal function was estimated in 38 patients who had both kidneys. Relative function of the right kidney $(\% R K)$ was calculated by the following equation:

$$
\% R K=100 \times(C r / A F r) /(C r / A F r+C r / A F l) .
$$

Differences in MAG3 clearance and relative function of the right kidney were calculated in each patient as the value derived using the $15 \%$ window minus that derived using the $20 \%$ window.

\subsection{Visual Evaluation of Image Quality}

The qualities of the perfusion image set (initial 203 -s images) and functional and excretory image set (30 1-min images) were visually compared between the $20 \%$ and $15 \%$ energy windows. Comparison was made independently by two board-certified nuclear medicine physicians, and discrepancy was resolved by consensus between them. The image sets obtained using the $20 \%$ and $15 \%$ energy windows in a given patient were displayed side-by-side on a monitor. The observers were blinded to the energy window width used to acquire each image set. They reviewed all images set, and assigned a score as follows: score 1, the left image set is superior to the right image set; score 2, both image sets are of equal quality; score 3, the left image set is inferior to the right image set.

\subsection{Statistical Analysis}

Data were expressed as mean $\pm \mathrm{SD}$. Linear regression analysis was made by the least squares method.

\section{Results}

The kidney ROI determined by the semiautomated method was modified manually for seven kidneys of five patients, among 78 kidneys of 40 patients. The need for manual modification was attributed to severely impaired function in three kidneys of three patients and large respiratory motion in four kidneys of two patients.

The MAG3 clearances using the $20 \%$ and $15 \%$ energy windows were $214.9 \pm 70.1 \mathrm{~mL} / \mathrm{min} / 1.73 \mathrm{~m}^{2}$ (range, $54.0-345.6 \mathrm{~mL} / \mathrm{min} / 1.73 \mathrm{~m}^{2}$ ) and $215.7 \pm 70.1 \mathrm{~mL} / \mathrm{min} / 1.73 \mathrm{~m}^{2}$ (range, $55.0-349.6 \mathrm{~mL} / \mathrm{min} / 1.73 \mathrm{~m}^{2}$ ), respec- 
tively. They showed close correlation with a correlation coefficient of 0.999 (Figure 1). The difference in MAG3 clearance was $0.8 \pm 2.3 \mathrm{~mL} / \mathrm{min} / 1.73 \mathrm{~m}^{2}$ (range, $-3.2-6.4 \mathrm{~mL} / \mathrm{min} / 1.73 \mathrm{~m}^{2}$ ).

The relative functions of the right kidney using the $20 \%$ and $15 \%$ energy windows were $51.4 \% \pm 16.1 \%$ (range, $8.7 \%-97.9 \%$ ) and $51.4 \% \pm 16.1 \%$ (range, $8.7 \%-98.0 \%$ ), respectively, in 38 patients having two kidneys. They showed close correlation with a correlation coefficient of 1.000 (Figure 2). The difference in \%RK was $0.0 \% \pm 0.1 \%$ (range, $-0.4 \%-0.2 \%$ ).

Visual evaluation indicated equal qualities for both perfusion image sets and functional and excretory image sets of all patients. There were no disagreements between the two observers for any result.

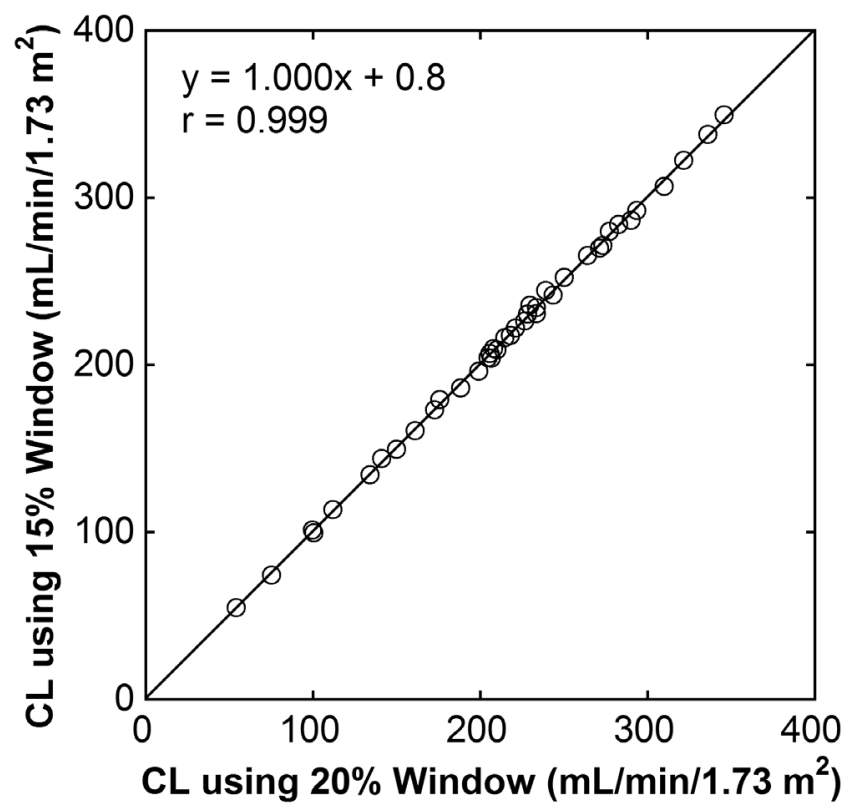

Figure 1. Relationship of MAG3 clearance (CL) between 20\% and $15 \%$ energy windows.

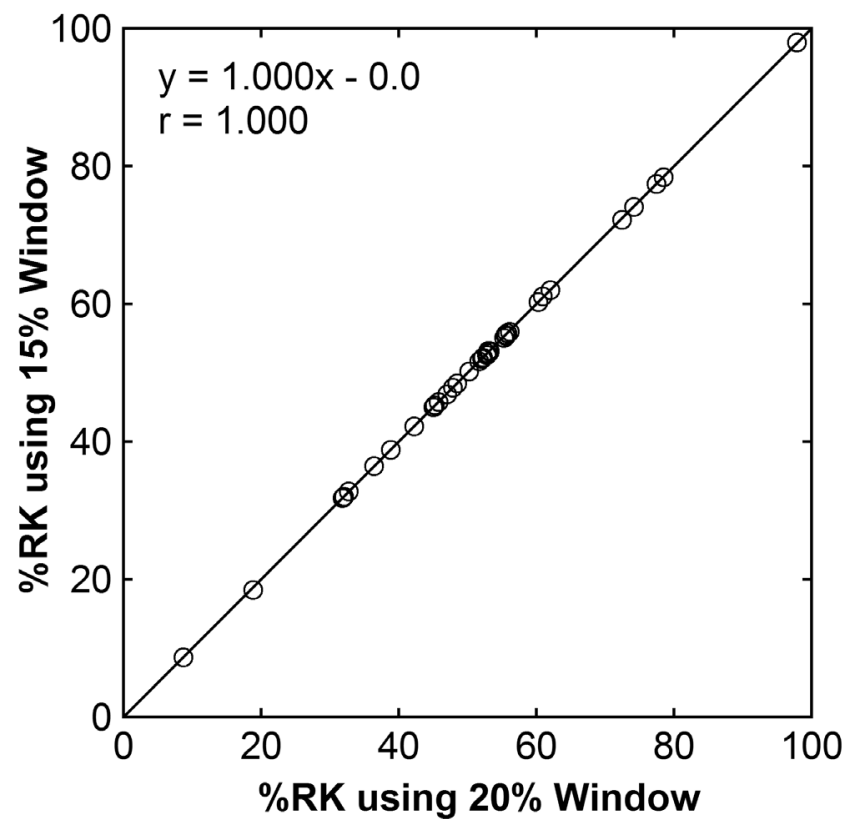

Figure 2. Relationship of relative function of the right kidney (\%RK) between $20 \%$ and $15 \%$ energy windows. 


\section{Discussion}

Quantitative evaluation of differential renal function is important in dynamic renal scintigraphy. In camerabased methods of estimating renal function, fractional renal uptake (the ratio of initial renal uptake to injected dose) is converted to an index of renal function using a predefined equation [1]-[5]. The width of the energy window is an essential parameter to define data acquisition using a gamma camera and may influence image quality and quantitative accuracy. In the present study, we investigated the effect of selection of the energy window width on the estimation of renal function from dynamic renal scintigraphy with ${ }^{99 \mathrm{~m}}$ Tc-MAG3. The gamma camera system used in this study can acquire images with distinct and overlapping energy windows simultaneously, facilitating precise comparison among different energy window widths.

The present study demonstrated that both total kidney MAG3 clearance and relative function of the right kidney were essentially identical between $20 \%$ and $15 \%$ energy windows, as indicated by concordant means, perfect correlations, and quite limited differences. The empirical equation employed was established using clinical data obtained with a $20 \%$ energy window [4]. Dynamic renal scintigraphy may be performed using a $20 \%$ or $15 \%$ energy window. Our results indicate that the same equation is applicable for the estimation of renal function from renal scintigraphy performed using a $15 \%$ energy window and that data obtained using $20 \%$ and $15 \%$ windows are interchangeable. The image quality was also similar irrespective of the energy window width. The selection of the energy window width of either $20 \%$ or $15 \%$ appears to be irrelevant for dynamic renal scintigraphy with ${ }^{99 \mathrm{~m}} \mathrm{Tc}-\mathrm{MAG} 3$.

\section{References}

[1] Taylor Jr., A., Corrigan, P.L., Galt, J., Garci, E.V., Folks, R., Jone, M., et al. (1995) Measuring Technetium-99mMAG3 Clearance with an Improved Camera-Based Method. The Journal of Nuclear Medicine, 36, 1689-1695.

[2] Itoh, K., Nonomura, K., Yamashita, T., Kanegae, K., Murakumo, M., Koyanagi, T., et al. (1996) Quantification of Renal Function with a Count-Based Gamma Camera Method Using Technetium-99m-MAG3 in Children. The Journal of Nuclear Medicine, 37, 71-75.

[3] Inoue, Y., Ohtake, T., Yokoyama, I., Yoshikawa, K., Asai, S. and Ohtomo, K. (1999) Evaluation of Renal Function from ${ }^{99 \mathrm{~m}}$ Tc-MAG3 Renography without Blood Sampling. The Journal of Nuclear Medicine, 40, 793-798.

[4] Inoue, Y. and Ohtomo, K. (2011) Estimation of Tc-99m MAG3 Clearance Using Camera-Based Methods without Blood Sampling. Clinical Nuclear Medicine, 36, 1012-1016. http://dx.doi.org/10.1097/RLU.0b013e31821a2a96

[5] Oriuchi, N., Onishi, Y., Kitamura, H., Inoue, T., Tomaru, Y., Higuchi, T., et al. (1998) Noninvasive Measurement of Renal Function with ${ }^{99 \mathrm{~m}}$ Tc-MAG3 Gamma-Camera Renography Based on the One-Compartment Model. Clinical Nephrology, 50, 289-294.

[6] Buvat, I., De Sousa, M.C., Di Paola, M., Ricard, M., Lumbroso, J. and Aubert, B. (1998) Impact of Scatter Correction in Planar Scintimammography: A Phantom Study. The Journal of Nuclear Medicine, 39, 1590-1596.

[7] Sjörgreen, K., Ljungberg, M. and Strand, S.E. (1996) Parameters Influencing Volume and Activity Quantitation in SPECT. Acta Oncologica, 35, 323-330. http://dx.doi.org/10.3109/02841869609101649

[8] Inoue, Y., Yoshikawa, K., Yoshioka, N., Watanabe, T., Saegusa, S., Kaneko, Y., et al. (2000) Evaluation of Renal Function with ${ }^{99 \mathrm{~m}} \mathrm{Tc}-\mathrm{MAG} 3$ Using Semiautomated Regions of Interest. The Journal of Nuclear Medicine, 41, 19471954.

[9] Inoue, Y., Yoshikawa, K., Suzuki, T., Katayama, N., Yokoyama, I., Kohsaka, T., et al. (2000) Attenuation Correction in Evaluating Renal Function in Children and Adults by a Camera-Based Method. The Journal of Nuclear Medicine, 41, 823-829. 